\title{
WIEWEIT SCHÜTZT DAS VÖLKERRECHT DEN AUSLANDISCHEN DIPLOMATEN IM FALLE SEINER ENTFÜHRUNG?
}

\author{
Von HermanN Weber
}

1.

Jahrhundertelang ruhte die Völkerrechtsordnung auf zwei Säulen: dem Diplomatenrecht, als der Ordnung der friedlichen Beziehungen, und dem Kriegsrecht, als der Ordnung der unfriedlichen Beziehungen zwischen den Staaten. Aber während das Kriegsrecht seinen fragmentarischen Charakter nie ganz verleugnen konnte, $j a$ in der jüngeren Epoche selbst fragwürdig geworden ist, hat das Diplomatenrecht, zuletzt in der Wiener Konvention von 1961 kodifiziert, eine Vervollkommnung erfahren, wie sie auf anderen Bereichen des internationalen Zusammenlebens, ungeachtet ihrer drängenden Regelungsbedürftigkeit, selten erreicht worden ist ${ }^{1}$.

Doch dieser Eindruck trügt. Denn die in jüngster Zeit zu beobachtende Praxis lateinamerikanischer Rebellen, ausländische Diplomaten ungeachtet deren Immunität gewaltsam zu entführen, um von der Regierung die Erfüllung bestimmter Forderungen zu erzwingen, ohne daß diese besondere Sicherungsvorkehrungen zugunsten der bedrohten Diplomaten trifft, stellt nicht lediglich eine Verletzung fundamentaler Völkerrechtsnormen dar, sondern hat die Diplomaten geradezu zum Instrument moderner Guerillakriegführung werden lassen. Damit drängt sich aber die Frage auf, ob der Schutz, den das geltende Völkerrecht den Repräsentanten eines fremden Staates im Ausland zuteil werden läßt, nicht an der Wirklichkeit "vorbeigarantiert" wird. Zumindest kann man sich angesichts der Vorkommnisse in Brasilien und Guatemala des Eindrucks nicht erwehren, daß das Völkerrecht dort, wo es seinen höchsten Entwicklungsstand erreicht zu haben scheint, eher dekorativ als überzeugend wirkt ${ }^{2}$.

Indessen wird man der neuen Situation, so tragisch sie im Einzelfall und so deprimierend sie im Hinblick auf die jahrelangen Bemühungen um eine Kodifizierung des Diplomatenrechts auch sein mag, nicht gerecht, wenn man sie nur als Faktum beklagt. Zu fragen ist vielmehr, wie der Tatbestand der Entführung ausländischer Diplomaten in das bestehende Völkerrechtssystem eingeordnet werden kann, damit ein bestmöglicher Schutz der offiziellen Vertreter der Staaten im Ausland erreicht wird. Diese Frage in ihrer ganzen Problematik zu erfassen, setzt jedoch die Kenntnis einiger Fakten voraus. Im folgenden sollen deshalb drei der spektakulärsten Entführungsfälle zunächst kurz vorgestellt werden:

a) Am 4. September 1969 wurde der amerikanische Botschafter in Brasilien, Charles Burke Elbrick, durch Mitglieder einer sich als ,Movimento Revolucinario 8 de Outobro ${ }^{\prime}{ }^{3}$ bezeichnenden Guerillaorganisation auf offener Straße in Rio de Janeiro entführt und 78 Stunden an einem geheimen Ort festgehalten, bis die Regierung die Forderungen der Rebellen erfüllte. Die brasilianische Regierung mußte, um das Leben des Botschafters zu schonen, fünfzehn von den Rebellen benannte politische Gefangene freilassen und nach Mexiko ins Exil verbringen. Sie mußte ferner ein Manifest der Guerillas über ihre Radio-

\footnotetext{
1 Der Konventionstext findet sich u. a. in den britischen Command Papers, Cmnd. 2565, in Auszügen auch bei B. Sen, A Diplomat's Handbook of International Law and Practice, The Hague 1965, S. 474 ff., und deutsch bei F. Berber, Völkerrecht, Dokumentensammlung, Bd. I, S. 865 ff.

2 So bereits Corbett, Law in Diplomacy, Princeton, N. J. 1959, S. 24.

3 Der Todestag Che Guevaras, vgl. Keesing's Contemporary Archives 23108.A.
} 
stationen verbreiten, in welchem zum Sturz der ,Diktatur' in Brasilien aufgerufen und die Entführung des Botschafters als ein ,Akt des ständig wachsenden revolutionären Befreiungskrieges' dargestellt wurde.

Die Annahme der von den Guerillas gestellten Bedingungen durch die Regierung Brasiliens war von Teilen der Streitkräfte heftig kritisiert worden. Außenminister Magelhães Pinto selbst erklärte, der von der amerikanischen Regierung ausgeübte Druck habe die Annahme der Forderungen der Rebellen unabweisbar gemacht $t^{4}$.

b) Am 31. März 1970 widerfuhr dem deutschen Botschafter in Guatemala, KarlMaria Graf von Spreti, in Guatemala-City das gleiche Schicksal. Nach seiner Entführung forderte eine Guerillaorganisation namens „Fuerzas Armadas Rebeldes" im Austausch für die Freilassung des Botschafters die Befreiung von sechzehn namentlich benannten politischen Gefangenen und deren Übergabe an die mexikanische Botschaft unter Zusicherung freien Geleits. Später erhöhten die Aufständischen ihre Forderung auf Freilassung von 22 Gefangenen und Zahlung eines Lösegeldes von 700000 US-Dollar. Doch spielte die Frage des Lösegeldes dann bei den Bemühungen um die Freigabe des Botschafters keine ausschlaggebende Rolle.

Die guatemaltekische Regierung indessen wollte sich nicht darauf verstehen, den Forderungen der Rebellen auf Freilassung bestimmter politischer Gefangener nachzukommen. Sie erklärte, daß es sich bei den von den Guerillas bezeichneten Personen um rechtskräftig Verurteilte handle, deren Freilassung in jedem Einzelfall nur durch das Appellationsgericht verfügt werden könne. Unbestätigten Gerüchten zufolge soll einer Freilassung aber auch der Umstand im Wege gestanden haben, daß sich unter den fraglichen Personen „vier Verräter" befanden, die eine Exekution seitens der Rebellen zu erwarten gehabt hätten.

Die mangelnde Bereitschaft der Regierung Guatemalas, die Forderungen der FAR zu erfüllen, veranlaßte die deutsche Bundesregierung, sich selbst um die Befreiung ihres Botschafters zu bemühen. Doch vermochten weder die persönlichen Appelle Bundeskanzler Brandts, noch die eindringlichen Warnungen Außenministers Scheels, noch die Entsendung des Sonderbotschafters Hoppe nach Guatemala, noch die Einschaltung offizieller und privater Mittelspersonen die guatemaltekische Regierung zum Einlenken zu bewegen. Darauf entschlossen sich die Guerillas, den in ihrer Gewalt befindlichen Botschafter zu töten.

Die deutsche Bundesregierung warf der Regierung Guatemalas vor, ihre völkerrechtlichen Schutzpflichten grob verletzt zu haben. Sie zeigte sich darüber um so mehr empört, als die guatemaltekische Regierung wenige Wochen zuvor auf die Forderungen der Rebellen eingegangen war, um ihren eigenen Außenminister auszulösen. Die Regierung Guatemalas begegnete diesem Vorwurf mit der Erklärung, daß die Gefangenen, die gegen Außenminister Fuentes Mohr ausgetauscht worden waren, noch nicht vor Gericht gestanden hätten ${ }^{5}$.

c) In der Nacht vom 11. auf 12. Juni 1970 stellte eine Untergrundorganisation dem deutschen Botschafter in Brasilien, Ehrenfried von Holleben, auf der Fahrt in seine Residenz einen Hinterhalt. Drei Sicherheitsbeamte, die den Botschafter seit der Ermordung Graf von Spretis auf allen seinen Wegen begleiteten, konnten die Entführung nicht verhindern. Die Rebellen verlangten von der brasilianischen Regierung wiederum im Austausch für den Botschafter die Freilassung politischer Gefangener, dieses Mal jedoch vierzig an der Zahl, die wahlweise nach Algerien oder Mexiko auszufliegen seien. Ferner forderten sie die Verbreitung eines revolutionären Manifests, für das

4 Keesing's 23706.A.

5 Vgl. Bulletin des Presse- und lnformationsamtes der Bundesregierung vom 21. 4. 1970 sowie Keesing's 23905.A. 
drei Guerillaorganisationen verantwortlich zeichneten ${ }^{6}$ und in dem es hieß: „Die Militärdiktatur demaskiere sich selbst immer mehr als Feind des Volkes und als Lakai der ausländischen Großkapitalisten." Als unmittelbare Ziele nannten die Rebellen in diesem Manifest u. a. den Sturz der Diktatur, Einsetzung einer Volkregierung, Abschaffung aller Akte und Dekrete der Diktatur, Enteignung der großen ausländischen Firmen und der Latifundien sowie die Ausrufung der Agrarrevolution.

Die Entführer rechtfertigten ihre Aktion vor der Offentlichkeit damit, daß alle Länder durch ihre Teilnahmslosigkeit am politischen Geschehen in Brasilien mitverantwortlich seien. Darüber hinaus könnten, so erklärten sie, diplomatische Vertreter von Ländern wie der Bundesrepublik, die „imperialistische Interessen“ in Brasilien vertreten, nicht als „neutral“ angesehen werden.

Die brasilianische Regierung erfüllte trotz der Widerstände, die ihr aus militärischen Kreisen entgegengesetzt wurden, die von den Guerillas gestellten Bedingungen, worauf Botschafter von Holleben wieder in Freiheit gesetzt wurde?

2.

Bei der Frage, welchen Schutz das Völkerrecht dem Diplomaten im Falle seiner Entführung in einem fremden Lande gewährt, ist vom Grundsatz der Integrität der Person des Diplomaten auszugehen, wie er sich als Folge einer jahrhundertealten, wenn auch nicht immer konsequenten Staatenpraxis als allgemeingültig herausgebildet und in Art. 29 der Wiener Konvention von 1961 seinen Niederschlag gefunden hat. Darin heißt es:

"The person of a diplomatic agent shall be inviolable. He shall not be liable to any form of arrest or detention. The receiving State shall treat him with due respect and shall take all appropriate steps to prevent any attack on his person, freedom or dignity." 8

In dieser ältesten aller diplomatischen Privilegien kristallisieren sich zwei Arten von Rechtspflichten des Empfangsstaates, aufgrund deren erst dem Diplomaten die Erfüllung der ihm übertragenen Aufgaben möglich ist: einmal die Pflicht, sich jeden $\mathrm{Zwanges}$ gegenüber dem Diplomaten zu enthalten, und zum anderen die Pflicht, den Diplomaten gegen jeden Angriff von privater Seite zu schützen?. Dies gilt auch für Brasilien und Guatemala, die zudem als Vertragsstaaten der Wiener Konvention unmittelbar aus Vertrag verpflichtet sind. Der von der Regierung Guatemalas vorgebrachte Einwand, sie habe auf die Bedingungen der Rebellen nicht eingehen können, weil es sich bei den freizulassenden Gefangenen um rechtskräftig Verurteilte gehandelt habe, war deshalb insoweit völkerrechtlich nicht relevant.

Nun hing aber in allen drei Entführungsfällen die Integrität des Diplomaten letztlich effektiv davon ab, ob die Regierung des Empfangsstaates bereit war, sich den erpresserischen Forderungen der Rebellen zu fügen. Die entscheidende Frage ist deshalb die, wie weit die Schutzpflicht einer Regierung reicht, insbesondere, ob sie sich in Maßnahmen erschöpft, die eine Entführung der bei ihr akkreditierten

\footnotetext{
6 Kommando Juarez Guimarães de Brito, Vangarda Popular Revolucionaria (VPR) und Acão Libertadora Nacional (ALN).

7 Vgl. Keesing's 24062.A.

8 S. o. Anm. 1.

9 Vgl. C. Hurst, Les immunités diplomatiques, Recueil des Cours, Bd. 12 (1926 II), S. $121 \mathrm{f} ., 124 \mathrm{ff}$.

Ph. Cahier und L. T. Lee, Vienna Conventions on Diplomatic and Consular Relations, International Conciliation Nr. 571, S. 20 ff., sowie B. Sen, a. a. O., S. 90 f.
} 
Diplomaten unmöglich machen, oder ob sie darüber hinaus auch solche Maßnahmen erfaßt, die, nachdem sich der allgemeine oder gar der besondere Schutz als unwirksam erwiesen hat, zur Wiederherstellung der Integrität des verletzten Diplomaten führen. Aus dem Wortlaut des Art. 29 der Wiener Konvention läßt sich diese Frage nicht mit Sicherheit beantworten. Denn die dort gewählte Formulierung „shall take all appropriate steps to prevent any attack" kann im restriktiven wie im extensiven Sinne ausgelegt werden. Es empfiehlt sich darum, das Problem von der Frage her anzugehen, ob sich aus dem Kontext dieses Abkommens in Verbindung mit den Vorarbeiten Umfang und Grenzen der dem Empfangsstaat obliegenden Schutzpflichten bestimmen lassen.

3.

Das Problem, wie weit der Schutz des fremden Diplomaten als Ausdruck seiner Immunität vor Angriffen auf seine Person, Freiheit und Würde reicht, ist durch den von A. E. F. Sandström der International Law Commission 1955 vorgelegten Entwurf der später abgeschlossenen Wiener Konvention deutlich zutage getreten. Danach sollte es den Behörden des Empfangsstaates erlaubt sein, vom Grundsatz der Unverletzlichkeit des Diplomaten abzugehen „in an extreme emergency, in order to eliminate a grave and imminent danger to human life, public health or property or to safeguard the security of the State ..."10. Die Diskussionen innerhalb der ILC darüber ergaben, daß darunter sowohl die Fälle einer akuten Gefährdung der Allgemeinheit verstanden wurden als auch solche, die, wie der Fall der diplomatischen Asylgewährung, in der Vergangenheit wiederholt die Frage nach den Grenzen der diplomatischen Unverletzlichkeit aufgeworfen hatten. Weder die ILC noch die Wiener Konferenz von 1961 konnten sich jedoch dazu entschließen, selbst in engen Ausnahmefällen vom Grundsatz der absoluten Integrität des Diplomaten abzugehen ${ }^{11}$. Dieses Prinzip, dem ursprünglich der Satz "par in parem non habet imperium" zugrundegelegen hat, das aber heute weniger als Vorrecht ad personam denn als notwendige Voraussetzung einer ungehinderten normalen Ausübung der diplomatischen Funktionen erklärt wird ${ }^{12}$, findet sich auch in anderen Bestimmungen der Konvention, so in Art. 22, wo die Unverletzlichkeit der Räumlichkeiten der diplomatischen Mission garantiert wird, ferner in Art. 24, wo die Archive und Dokumente der Mission als unverletzlich "at any time whereever they may be" bezeichnet werden, oder in Art. 45, wo die Mission mitsamt ihres Eigentums und der Archive selbst im Falle eines zwischen dem Empfangsstaat und dem Entsendestaat ausgebrochenen bewaffneten Konfliktes zu schützen sind.

Nun ist aber die Immunität, die den Diplomaten regelmäßig schützt, beileibe keine absolute. Solche genießt er im Bereich der Gerichtsbarkeit beispielsweise nur in Strafsachen. In Angelegenheiten der Zivil- und Verwaltungsgerichtsbarkeit hingegen ist seine Immunität nur eine beschränkte. So können nach Art. 31 der Wiener Konvention gegen den Diplomaten dingliche Klagen in bezug auf sein privates im Empfangsstaat belegenes unbewegliches Vermögen, Klagen in Nachlaßsachen und Klagen im Zusammenhang mit einer nebenamtlichen Tätigkeit angestrengt wer-

10 UN-Doc. A/CN. 4/91, Art. 12.

$11 \mathrm{Zu}$ den Diskussionen in der ILC s, Yearbook of the International Law Commission, 1957, Bd. 1, S. $54 \mathrm{ff}$., und 1958, Bd. 1, S. 127 ff. Zu den Diskussionen auf der Wiener Konferenz 1961 s. Conference on Diplomatic Relations, Bd. 1, S. $135 \mathrm{ff}$.

12 Vgl. I. P. Blisčenko und V. N. Durdenevskij, Diplomatičeskoe i konsul'skoe pravo, Moskva 1962, S. 335 ff., insbes. S. 343 . 
den ${ }^{12 a}$. Aber auch in diesen Fällen, so heißt es in Abs. 3 dieser Bestimmung, dürfen Vollstreckungsmaßnahmen die Unverletzlichkeit der Person des Diplomaten oder seiner Wohnung nicht beeinträchtigen.

Die Integrität der Person des Diplomaten erscheint also nach dem Kontext der Wiener Konvention vollkommen, soweit es das aktive Verhalten des Empfangsstaates gegenüber den bei ihm akkreditierten fremden Diplomaten angeht. Trifft diese Feststellung aber auch in solchen Fällen zu, in denen ohne direktes Zutun staatlicher Organe ein Diplomat zu Schaden kommt?

Nach Art. 29 der Wiener Konvention ergreift der Empfangsstaat alle geeigneten Maßnahmen, um jeden Angriff auf seine Person, seine Freiheit oder seine Würde zu verhindern. Der absolute Schutz, der in dieser Vorschrift gesehen werden kann, erfährt jedoch eine Einschränkung durch die weitere Erklärung, daß es sich hierbei nur um "geeignete“ Maßnahmen handeln kann.

Doch ist der unbestimmte Rechtsbegriff der Geeignetheit einer Maßnahme nicht unbeschränkt auslegungsfähig. Schließt eine authentische Interpretation des Textes auch die Möglichkeit aus, daß unter „geeigneten“ Maßnahmen „alle nur denkbaren" Maßnahmen verstanden werden können, so ist damit noch nicht gesagt, daß der Empfangsstaat nach freiem Belieben darüber befinden darf, welche Maßnahmen ihm subjektiv als geeignet dünken. Unter der Voraussetzung eines weitestgehenden und möglichst effektiven Diplomatenschutzes ist vielmehr die Annahme gerechtfertigt, daß der Empfangsstaat verpflichtet ist, alle Maßnahmen zu ergreifen, die objektiv geeignet sind, einen Angriff auf Person, Freiheit und Würde des Diplomaten $\mathrm{zu}$ verhindern. Ob diese Regel in irgendeiner Form eine Grenze findet, etwa im Grundsatz der Verhältnismäßigkeit, wird dabei noch zu erörtern sein. Hier soll zunächst nur die Feststellung genügen, daß die Pflicht, objektiv geeignete Maßnahmen zu treffen - und nur um solche kann es sich in Art. 29 handeln - dem Diplomaten einen weitaus wirksameren Schutz verleiht als die Pflicht zu nur subjektiv geeigneten Maßnahmen. Sie läßt den Empfangsstaat für Handlungen haften, die er nicht lediglich zu verhindern völkerrechtlich verpflichtet ist, sondern auch die zu verhindern er objektiv in der Lage ist.

4.

Sosehr dieses Ergebnis der Feststellung einer weitestgehenden Schutzpflicht des Empfangsstaates nahekommt, so erlaubt es doch noch nicht den sicheren Schluß, daß diese Pflicht dem Diplomaten gegenüber in jedem Fall zur Anwendung kommt. Denn das Ansinnen an den Empfangsstaat, den Diplomaten auch im Falle seiner Entführung zu schützen, stellt an dessen Schutzpflicht Anforderungen, die einer Erfolgshaftung gleichkommen. Ein Vergleich dieser besonderen Schutzpflicht gegenüber dem Diplomaten mit der allgemeinen Schutzpflicht, wie sie gegenüber allen Fremden zu beobachten ist, soll deshalb die Frage beantworten helfen, ob diese Rechtsfolge aus der besonderen Schutzpflicht des Empfangsstaates tatsächlich herausgelesen werden kann.

\footnotetext{
12a Vgl. hierzu den Beschluß des Bundesverfassungsgerichtes vom 30. 4. 1963, wonach keine allgemeine Regel des Völkerrechts bestehe, nach der die inländische Gerichtsbarkeit für Klagen gegen einen aus. ländischen Staat in bezug auf seine nicht-hoheitliche Betätigung ausgeschlossen sei, in: Entscheidungen des BVG, Bd. 16, S. 33. Vgl. auch G. Dahm, Völkerrechtl. Grenzen der inländischen Gerichts . barkeit gegenüber ausländischen Staaten, Festschrift f. A. Nikisch, Tübingen 1958, S. $163 \mathrm{ff}$.
} 
In der Völkerrechtslehre wird seit langem angenommen, daß Fremde gegen Verletzungen ihrer Person und ihres Vermögens angemessenen Rechtsschutz genießen (sogenannter völkerrechtlicher Mindeststandard) ${ }^{13}$. Daraus ergeben sich bestimmte Pflichten für den Aufenthaltsstaat, die den Mindestanforderungen genügen müssen, die in der Kulturwelt üblich sind und hinter denen der Aufenthaltsstaat gegenüber den Fremden nicht zurückbleiben darf. Man weiß sich ferner darüber einig, daß ein Staat für das Fehlverhalten seiner Organe einzustehen hat, und zwar sowohl für das direkte Fehlverhalten als auch dafür, daß seine Organe es unterlassen haben, gegen schädigende Handlungen Privater einzuschreiten. Ein Staat haftet deshalb auch für die auf seinem Gebiet begangenen Handlungen von Privatpersonen, seien diese Staatsangehörige oder Staatsfremde, wenn er die Vornahme der Handlungen nicht verhindert oder die Bestrafung unterlassen hat, obgleich er dazu in der Lage gewesen wäre. Ein solches Unterlassen wird in seinem Unrechtsgehalt einem aktiven Tun gleichgestellt ${ }^{14}$.

Dabei macht es keinen rechtlichen Unterschied, ob die inkriminierende Handlung durch gewöhnliche Privatpersonen oder durch Rebellen vorgenommen wurde, die mit ihr politische Ziele verfolgen. Da Rebellen keine Organe des Staates sind, für deren Handlungen dieser nach den allgemeinen Grundsätzen über die Staatshaftung verantwortlich ist, andererseits sie auch nicht selbständiges Völkerrechtssubjekt sind, solange sie nicht von der eigenen Regierung oder der eines dritten Staates als Kriegführende anerkannt werden, und sie damit auch nicht aus eigenem Rechte haftungsfähig sind, haftet für ihre Handlungen der Staat wie für Einzelpersonen ${ }^{15}$. Aus diesem Grunde hat auch Sandström als Berichterstatter der International Law Commission in seinem revidierten Entwurf einer Konvention über die Staatenhaftung die Haftung des Staates für das Verhalten von Privatpersonen im Falle von Unruhen mit der im Normalfall gegebenen Verantwortlichkeit des Staates gleichgestellt und einer einheitlichen Regelung unterworfen ${ }^{16}$.

Kontrovers hingegen ist seit längerer Zeit die Frage, ob Schuld Voraussetzung der Haftung des Staates ist. In der Lehre stehen sich die Theorien der Schuld- und Erfolgshaftung unversöhnlich gegenüber. Während noch die auf Grotius zurückgehende traditionelle Schuldtheorie einen Staat für Handlungen seiner Organe oder Bürger ohne eigenes Verschulden völkerrechtlich nicht haften läß $\mathrm{t}^{17}$, hat mit den Lehren Triepels und Anzilottis die Theorie der Erfolgshaftung zunehmende Verbreitung gefunden, ohne indessen die Schuldtheorie $\mathrm{zu}$ verdrängen ${ }^{18}$.

13 Vgl. G. Dahm, Völkerrecht, Bd. 1, Stuttgart 1958, S. 503 ff., und Wörterbuch des Völkerrechts (Strupp-Schlochauer), Bd. 2, S. 537 f. A. A. die sowjetische Doktrin, vergl. D. B. Levin, Die Verantwortlichkeit der Staaten im gegenwärtigen Völkerrecht, Potsdam-Babelsberg 1969, S. $148 \mathrm{ff}$.

$14 \mathrm{~S}$. C. Eagleton, The Responsibility of State in International Law, New York 1928, S. 125 ff. und 182 ff., I. von Münch, Das völkerrechtliche Delikt in der modernen Entwicklung der Völkerrechtsgemeinschaft, Frankfurt (M.) 1963, S. 163, 225 und 228 mit weiteren Nachweisen, und D. B. Levin, a. a. O., S. 112. Vgl. auch die dort zitierten Kodifikationsentwürfe des Institut de Droit 1927, der Haager Kodifikationskonferenz 1930 und der Deutschen Gesellschaft für Völkerrecht 1930. Ferner: Second Report on State Responsibility, UN-Doc. A/CN. 4/233, S. $28 \mathrm{ff}$.

15 Vgl. G. Dahm, Völkerrecht, Bd. 3, Stuttgart 1961, S. 200 ff. Aus der älteren Lehre P. Schoen, Die völkerrechtliche Haftung der Staaten aus unerlaubten Handlungen, Dresden 1917, S. 79 f., K. Strupp, Das völkerrechtliche Delikt, Stuttgart 1920, S. 89 ff., J. Spiropoulos, Die De-facto-Regierung im Völkerrecht, Kiel 1926, S. 183 ff.

16 Yearbook of the ILC, 1961, Bd. II, S. 46, Art. 7.

17 Hugo Grotius, De jure belli ac pacis Libri tres. XVIII, XX, 1, 2, G. Dahm, a. a. O., S. 228 f., Oppenheim-Lauterpacht, International Law, Bd. 1, 8. Aufl., S. 343, A. Verdross, Völkerrecht, 5. Aufl. Wien 1964, S. $393 \mathrm{ff}$.

18 Vgl. H. Triepel, Völkerrecht und Landesrecht, S. 328 ff., D. Anzilotti, Teoria generale della responsibilita dello stato nel diritto internazionale, Florenz 1902, S. 153 ff., P. Guggenheim, Völkerredit, Bd. 2, Basel 1951, S. 556 ff. Ausführlich hierzu I. von Münch, a. a. O., S. 152 ff. 
Der Internationale Gerichtshof in Den Haag hat seinerseits sich nicht dazu entschließen können, einer der beiden Ansichten den Vorzug zu geben ${ }^{19}$. Auch eine allgemeine Betrachtung der Staatenpraxis läßt nicht den Schluß zu, diese habe sich mit Bestimmtheit für oder gegen eine der beiden Theorien ausgesprochen ${ }^{20}$.

Deshalb ist die Frage, ob ein deliktisches Verhalten nur bei Schuld gegeben ist oder ob bereits der Erfolgseintritt genügt, für das Völkerrecht nicht generell, sondern für jeden Einzelfall des deliktischen Verhaltens gesondert zu beantworten ${ }^{21}$. Die Staaten aber haben in den Fällen, in denen Fremde durch Unruhen zu Schaden gekommen waren, regelmäßig eine Haftung abgelehnt, wenn nicht ein konkretes Verschulden staatlicher Organe nachgewiesen wurde. Soweit Fremde für erlittene Schäden dennoch entschädigt wurden, geschah dies allein aus politischer Rücksichtnahme 22. Nach südamerikanischer Auffassung sollte in derartigen Fällen eine Verantwortlichkeit des Aufenthaltsstaates sogar nur dann eintreten, wenn sich der Aufstand gerade gegen die Fremden richtete und der Staat hiergegen pflichtwidrig nicht eingeschritten ist ${ }^{23}$.

Die neueren Kodifikationsbemühungen der UNO gehen dahin, den Staat nur bei Vorliegen von Fahrlässigkeit für das Unterlassen seiner Organe haften zu lassen. So heißt es in dem von Garcia Amador ausgearbeiteten Entwurf einer Staatenhaftung: „The State is responsible for injuries caused to aliens by acts of ordinary private individuals, if the organs or officials of the State where manifestly $\mathrm{negligent} \mathrm{t}^{24}$ in taking the measures which are normally taken to prevent or punish such acts“25. Mit der Aufnahme der "negligence“ aber ist die Schuld zu einem Kriterium der Staatshaftung gemacht worden ${ }^{26}$, wenngleich nicht zu verkennen ist, daß der Begriff der "negligence“, der dem angelsächsischen Recht entnommen ist, nicht zweifelsfrei mit dem römischrechtlichen „culpa“-Begriff identifiziert werden kann ${ }^{27}$. Im Falle der Verletzung gewöhnlicher Fremder durch Handlungen Privater wird man daher davon ausgehen können, daß die Staaten auch in Zukunft am Erfordernis der Schuld als Haftungsvoraussetzung festhalten werden. Die Schuld macht es nicht nur abstrakt möglich, eine nicht eindeutige Vökerrechtsregel im Zweifelsfalle restriktiv auszulegen, sondern sie dient auch als Korrektiv, um schwierige Umstände, in die staatlichen Organe in Zeiten innerer Unruhen leicht geraten, bei der Haftungsfrage angemessen zu berücksichtigen ${ }^{27 a}$.

19 Vgl. den Korfu-Kanal-Fall, ICJ Reports 1949, S. 4 ff.

20 Beispiele bei G. Dahm, a. a. o., S. $226 \mathrm{ff}$.

21 So I. von Münch, a. a. O., S. 161.

$22 \mathrm{Vgl}$. J. B. Moore, A. Digest of International Law, Bd. 6, Washington 1906, S. $956 \mathrm{ff}$. Konsequent in diesem Zusammenhang ist auch die Reaktion der deutschen Bundesregierung auf die Entführung zweier deutscher Ingenieure durch bolivianische Guerillas im Juli 1970 gewesen. Anders als im Falle der Botschafterentführungen hat die Bundesregierung sich jeder völkerrechtlichen Argumentation enthalten und den bolivianischen Staatspräsidenten nur gebeten, sich persönlich um die Angelegenheiten zu kümmern. Als die Regierung Boliviens die Forderung der Rebellen auf Freilassung von zehn politischen Häftlingen erfüllt hatte, dankte ihr die Bundesregierung dafür, daß sie die "Gebote der Menschlichkeit vor alle innenpolitischen Uberlegungen gestellt habe ${ }^{4}$, vgl. Die Welt vom 23. und 25. 7. 1970.

23 So Ziff. 8 des Berichtes Guerreros auf der Haager Kodifikationskonferenz 1930, abgedruckt bei C. Eagleton, a. a. O., S. 235 (259).

24 Hervorhebung durch den Verfasser.

25 Yearbook of the ILC, 1957, Bd. II, S. 120, Art. 10. Ebenso Art. 11 für den Fall von Aufständen, Bürgerkrieg und inneren Unruhen.

26 Art. 10 des Harvard-Entwurfes 1929 nennt erstmals die "due diligence“ als Haftungsvoraussetzung, vgl. American Journal of International Law, Bd. 23 (1929), S. $133 \mathrm{ff}$.

27 Vgl. dazu G. Dahm, a. a. O., S. 224 Anm. 2, I. von Münch, a. a. O., S. 162. Garcia Amador allerdings bezeichnete in seinem Kommentar zu Art 10-12 die "due diligence" als die "expression par excellence of the so-called theory of fault (culpa) “, vgl. Yearbook of the ILC, 1957, Bd. II, S. 122.

27 a Der neueste Report on State Responsibility vom 20. 4. 1970 geht ebenfalls ganz allgemein von der Existenz eines objektiven und eines subjektiven Elementes bei der Feststellung eines internationalen Deliktes aus, a. a. O., S. $25 \mathrm{ff}$ 
Schon dieser Vergleich der allgemeinen Schutzpflicht mit der besonderen, wie sie gegenüber fremden Diplomaten zu beobachten ist, zeigt, daß die Überlegungen, die zu einer Haftungsbegrenzung des Aufenthaltsstaates im Falle der Verletzung Fremder führen, nicht auf den Fall der Haftung des Empfangsstaates für die Verletzung bei ihm akkreditierter Diplomaten übertragen werden können. Die Haftung des Empfangsstaates für die Handlungen Privater auf den Fall des nachgewiesenen Verschuldens staatlicher Organe zu limitieren, hieße dem akkreditierten Diplomaten nur den Standard an Rechten zuzuerkennen, wie er jedem Fremden im Aufenthaltsstaat zusteht. Im Falle seiner Entführung durch Rebellen könnte demnach der Diplomat nur beanspruchen, nicht schlechter gestellt zu werden als jeder andere Fremde auch. Sein Fall würde sich in nichts von anderen Entführungsfällen unterscheiden, denen die Strafverfolgungsbehörden ex officio nachzugehen haben. $\mathrm{Zu}$ weitergehenden Schutzmaßnahmen wäre der Empfangsstaat nicht verpflichtet.

Tatsächlich hat es auch Stimmen gegeben, die dem Diplomaten keinen weiterreichenden Schutz zubilligen wollten als den, der allen Bewohnern des Staates, Staatsfremde eingeschlossen, nach den Gesetzen des Landes zugestanden wird ${ }^{28}$. Seit langem ist es jedoch herrschende Ansicht, daß der Empfangsstaat gegenüber den bei ihm akkreditierten Diplomaten einer erhöhten Schutzpflicht unterliegt, einer Pflicht, die, wie Bluntschli es bereits formuliert hat, „zugunsten des directen Völkerverkehrs mit Bezug auf die Gesandten gesteigert und gleichsam potenziert wird“29. Der aus Anlaß des Korfu-Zwischenfalls eingesetzte juristische Expertenausschuß des Völkerbundes hat 1924 ausdrücklich festgestellt: „Le caractère public reconnu que revêt un étranger, les circonstances dans lesquelles il se trouve sur le territoire de l'Etat entraînent, pour celui-ci, un devoir de vigilance spéciale à son égard"30. Um dieser besonderen Verpflichtung gerecht zu werden, haben auch zahlreiche Staaten Spezialstraftatbestände für den Fall der Verletzung der Diplomaten geschaffen ${ }^{31}$. In dieser Steigerung der Schutzpflicht im Falle der Diplomaten liegt die Vermutung begründet, daß der Diplomat einen stärkeren Schutz beanspruchen darf, weil er, anders als der Fremde ganz allgemein, für die Aufrechterhaltung der zwischenstaatlichen Beziehungen unmittelbar verantwortlich ist. Andernfalls könnte der Diplomat, der nur den Schutz zu beanspruchen berechtigt wäre, der allenfalls zu einer Haftung bei nachgewiesenen Verschulden des Empfangsstaates führt, seinen Aufgaben nicht gerecht werden ${ }^{31 a}$. Während also die Schuldhaftung bei Verletzung Fremder durch Privatpersonen grundsätzlich noch zu angemessenen Ergebnissen führen kann, würde sie im Falle der Diplomaten dem Wesen des Gesandschaftsrechtes eklatant widersprechen. Die echte Erfolgshaftung ist daher im Falle der Entführung eines akkreditierten Diplomaten die

$28 \mathrm{Vgl}$. A. B. Lyons, Personal Immunities of Diplomatic Agents, British Yearbook of International Law, Bd. 31 (1954), S. $304 \mathrm{f}$.

$29 \mathrm{Vgl}$. Das moderne Völkerrecht der civilisierten Staten, Nördlingen 1872, Art. 192.

30 Société des Nations, Journal Officiel, 1924, S. 524. Ebenso der Kommentar zu Art. 17 des HarvardEntwurfes von 1932, vgl. American Journal of International Law, Bd. 26 (1932), S. 96, C. Hurst, a. a. O., S. 124, Ph. Cahier und L. T. Lee, a. a. O., S. 23 und 26, E. Satow, A Guide to the Diplomatic Practice, 4. Aufl. London 1957, S. 176, B. Sen, a. a. O., S. 90.

31 Vgl. $\oint 102$ des deutschen Strafgesetzbuches. Für die anderen Staaten s. Laws and Regulations Regarding Diplomatic and Consular Privileges and Immunities, ST/LEG/SER.B/7.

31a Welche Bedeutung die Staaten dem Schutz des Diplomaten beimessen, ist auch dem Schreiben zu entnehmen, das der ständige Vertreter der Niederlande bei den Vereinten Nationen an den Präsidenten der UN-Vollversammlung gerichtet hat und in dem es heißt, ein Angriff auf die Person des Diplomaten könnte einen Streit auslösen, als dessen Folge der Weltfrieden und die internationale Sicherheit gefährdet sei, vgl. UN-Doc. A/CN. 4/235, Annex (Schreiben vom 5. 5. 1970). 
allein adäquate Haftungsform, zumal sie als die strengere Haftung den Empfangsstaat zu erhöhter Sorgfalt bei der Vermeidung eines solchen Angriffs zwingt. Der Diplomat darf im Falle seiner Entführung erwarten, daß der Empfangsstaat alles in seiner Macht Stehende unternimmt, um seine Integrität wiederherzustellen.

Die Feststellung, daß der Empfangsstaat für die Verletzung bei ihm akkreditierter Diplomaten in jedem Falle, also auch ohne Verschulden seiner Organe haftet, weil er ihnen gegenüber einer erhöhten Schutzpflicht unterworfen ist, findet ihre Parallele in den neueren internationalen Haftungsübereinkommen, in denen die Tendenz der Staaten erkennbar wird, in allen Fällen eines erhöhten Gefahrenrisikos die Gefährdungshaftung an die Stelle der Schuldhaftung treten zu lassen. So hat die Gefährdungshaftung als beherrschende Haftungsform Eingang gefunden in das Brüsseler Abkommen über die Haftung für Schiffe mit Nuklearantrieb von 1962, in den Weltraumvertrag von 1967 und in das Brüsseler Abkommen über die zivilrechtliche Haftung für Olverschmutzungsschäden von 196932. Ferner statuieren alle nationalen Rechte auf dem Gebiet der Kernenergieschäden die Gefährdungshaftung, die auch in die entsprechende Konvention der OEEC übernommen worden ist ${ }^{33}$. Ingo von Münch erklärt aus diesem Grunde, daß möglicherweise insoweit bereits von einem allgemeinen Rechtsgrundsatz im Sinne des Art. 38 Ziff. 1 lit. c des Statuts des Internationalen Gerichtshofes gesprochen werden $\mathrm{kann}^{34}$.

6.

Ist nun der Empfangsstaat gegenüber den bei ihm akkreditierten Diplomaten grundsätzlich unbeschränkt schutzpflichtig, so folgt daraus für die eingangs erwähnten Entführungsfälle, daß die Regierungen Brasiliens und Guatemalas nach dem Völkerrecht verpflichtet waren, nicht nur die Entführung der Botschafter zu verhindern, sondern darüber hinaus auch alle Maßnahmen zu ergreifen, die objektiv geeignet waren, den entführten Botschaftern die Freiheit wiederzugeben. Die Botschafter Elbrick, von Spreti und von Holleben hatten somit einen Rechtsanspruch darauf, daß die Regierung der betroffenen Gaststaaten die Forderungen der Rebellen erfüllte, weil dadurch ihre Wiederbefreiung objektiv erlangt werden konnte. Sie hatten diesen Rechtsanspruch um so mehr, als alle Versuche, der Rebellen habhaft zu werden, um die entführten Botschafter gewaltsam zu befreien, sich sehr schnell als unwirksam erwiesen hatten.

Hier jedoch stößt sich das Recht an der Wirklichkeit, denn ein perfekter Schutz der Diplomaten wird sich in praxi niemals erzielen lassen, jedenfalls dort nicht, wo innenpolitische Kämpfe auf dem Rücken der Diplomaten ausgetragen werden ${ }^{35}$. Auf der anderen Seite bedingt aber die erhöhte Schutzpflicht des Empfangsstaates verschärfte Sicherheitsbestimmungen für die gefährdeten Botschaften. Wo aber auch solche die Entführung nicht verhindern konnten, wobei durchaus zweifelhaft erscheint, ob in jedem Einzelfall nach dem ersten bekanntgewordenen Entführungsfall zusätzliche Vorkehrungen getroffen wurden und ob diese als ausreichend anzu-

\footnotetext{
32 Vgl. International Legal Materials, Current Documents, 1967, S. 386, Conférence Diplomatique de Droit Maritime, $11 \mathrm{me}$ Session, Brüssel 1962, S. 720, und International Legal Materials, a. a. O., 1969, S. 453.

33 Vgl. Art. 3 der Konvention vom 29. 7. 1960, American Journal of International Law, Bd. 55 (1961), S. 1082.

34 A. a. O., S. 168.

35 So auch Außenminister Scheel, vgl. Süddeutsche Zeitung vom 13./14. 6. 1970.
} 
sehen waren, da stellt sich die Frage, wo die Grenze der politischen und rechtlichen Zumutbarkeit erreicht ist, jenseits der das Verlangen auf Erfüllung der von den Rebellen erhobenen Forderungen rechtsmißbräuchlich wird.

Ein Staat ist regelmäßig nach dem Völkerrecht nicht verpflichtet, den Schutz der unter seine Obhut gestellten Personen unter Bedingungen zu gewährleisten, die ihm ein unverhältnismäßiges Opfer auferlegen würden. Im Völkerrecht ist der Grundsatz der Verhältnismäßigkeit der angewandten Mittel und des verfolgten Zweckes von jeher ein alle Normen beherrschendes Rechtsprinzip. Alle Maßnahmen sind daher auf das Maß dessen beschränkt, was noch als verhältnismäßig zum behaupteten Unrecht betrachtet werden $\mathrm{kann}^{36}$. So ist beispielsweise allgemein anerkannt, daß Selbstverteidigungsmaßnahmen nicht außerproportional zum angerichteten Schaden stehen dürfen. Die Tatsache, daß die Anwendung dieses Rechtsprinzips wegen seiner begrifflichen Ungenauigkeit in Einzelfällen fraglich erscheinen kann, führt dabei nicht zu der Vermutung, es sei rechtlich überhaupt nicht relevant ${ }^{37}$.

Für die besondere Schutzpflicht des Empfangsstaates gegenüber den bei ihm akkreditierten ausländischen Staatenvertretern gilt nichts anderes. In diesem Fall der gesteigerten Haftung liegt nur die Schwelle höher, jenseits der das dem Empfangsstaat abverlangte Opfer unverhältnismäßig und damit unzumutbar wird. Die Selbstaufgabe des Staates beispielsweise wäre eine Forderung, der keine Regierung auch unter dem Gesichtspunkt des absoluten Diplomatenschutzes nachzukommen verpflichtet sein $\mathrm{kann}^{38}$. War diese Opferschwelle aber im Falle der Entführung der drei Botschafter Elbrick, von Spreti und von Holleben überschritten?

Allen drei Entführungsfällen gemeinsam war die Forderung der Rebellen, eine bestimmte Anzahl politischer Häftlinge freizulassen. In zwei Fällen wurde darüber hinaus die Verbreitung revolutionärer Proklamationen über den Informationsapparat der Regierung verlangt, während die in einem Fall geforderte Zahlung eines Lösegeldes nicht unabdingbar war. Keine dieser Forderungen hatte den betroffenen Regierungen ein Opfer auferlegt, das ihnen nach dem Proportionalitätsgrundsatz nicht hätte zugemutet werden können. Auch wenn als Folge der Ausstrahlung der politischen Proklamationen der Rebellen über die Radiosender der Regierung mit Unruhen in der Bevölkerung zu rechnen gewesen wäre oder wenn zu erwarten gestanden hätte, daß die politischen Gefangenen unmittelbar nach ihrer Freilassung in ihrer konspirativen Tätigkeit fortfahren würden, hätte eine Erfüllung der Forderungen der Rebellen mit Sicherheit noch im Rahmen des Zumutbaren gelegen, wie das Nachgeben der brasilianischen Regierung ja deutlich beweist. Dasselbe gilt für die Befürchtungen, die in den Militärkreisen der betroffenen Staaten gehegt worden waren, wonach die Entführungen durch ein Nachgeben der Regierung erst voll wirksam würden und die Rebellen erst dadurch eine Ermunterung zu neuen Entführungsaktionen erführen. Denn nicht eigentlich diese Gefahr, sondern der Umstand, daß die betroffenen Gaststaaten sich selbst als unfähig erwiesen haben, den ihnen anvertrauten Diplomaten den gebührenden

36 Vgl. das Schiedsurteil im Naulilaa-Fall, Reports of International Arbitral Awards II, S. $1025 \mathrm{ff}$

37 Vgl. hierzu Ian Brownlie, International Law and the Use of Force by States, Oxford 1963, S. $261 \mathrm{ff}$. A. A. R. R. Baxter, The Legal Consequences of the Unlawful Use of Force under the Charter, Proceedings of the American Society of International Law, 1968, S. 74.

38 Ebenso I. von Münch in Die Zeit vom 26. 6. 1970. 
erhöhten Schutz zu gewährleisten, hat den Grad dessen bestimmt, was ihnen als Opfer für die Wiederherstellung der Integrität der Diplomaten zugemutet werden konnte.

Das Maß der Zumutbarkeit im Rahmen einer bestehenden Restitutionspflicht hängt also wesentlich vom Grad der Vernachlässigung der Schutzpflichten des Empfangsstaates ab. So war die der brasilianischen Regierung auferlegte Opfergrenze im Falle der Entführung des Botschafter von Holleben besonders hoch anzusetzen, weil Art und Umfang der dem deutschen Missionschef beigeordneten Bedeckung unter den gegebenen Umständen keinen der Gefahr einer Entführung, mit der man konkret gerechnet hatte, adäquaten Schutz boten. Die brasilianischen Behörden hatten zwar drei Bewacher zum Schutze von Hollebens abgeordnet, von denen einer im Dienstwagen des Botschafters und zwei in einem nachfolgenden Lieferwagen der Botschaft saßen. Aber weder die Fahrzeuge noch das Ausmaß der Bewachung haben einen angemessenen Schutz gegen einen Überfall geboten, zumal die Behörden nicht bedacht hatten, daß die örtlichen Umstände nach Einbruch der Dunkelheit einen Überfall geradezu begünstigen mußten. Die Wochenzeitschrift „Die Zeit" schrieb bereits lange vor der Entführung von Hollebens: „Die serpentinenreiche Auffahrt zur Residenz ist wie geschaffen für einen Hinterhalt. Und ob die drei "Gorillas" im Volkswagen eine entschlossene Guerillaattacke abwehren könnten, bleibt mindestens zweifelhaft."39 Außenminister Scheel selbst hatte die Sicherungsmaßnahmen für den deutschen Botschafter für „wahrscheinlich ausreichend" gehalten und daran die Frage geknüpft: „Soll er mit einem Panzer begleitet werden?"40 Doch ist die Bewachung eines Diplomaten durch ein Panzerfahrzeug nicht die einzige und zudem eine höchst fragwürdige Alternative ${ }^{41}$.

Dagegen würde die Erfüllung der Forderung der Rebellen auf Auslieferung sogenannter „Verräter“, um diese im Wege der Selbstjustiz zu liquidieren, das Maß dessen überschreiten, was dem Empfangsstaat auch unter dem Gesichtspunkt einer hoch anzusetzenden Opfergrenze noch als zumutbares Opfer abverlangt werden könnte. Kein Staat ist verpflichtet, den Schutz der Diplomaten auf Kosten der Menschlichkeit zu gewährleisten. Dies betont schon die Allgemeine Erklärung der Menschenrechte, wonach „alle Menschen frei und gleich an Würde und Rechten geboren sind" und "jeder Mensch Anspruch auf Rechtsschutz hat"42. Das Leben eines Diplomaten ist insoweit nicht schutzwürdiger als das eines politischen Gefangenen $^{43}$.

Im Falle der Entführung des Botschafters Graf von Spreti war jedoch die Sorge, die Rebellen wollen gleichzeitig mit der Forderung auf Freilassung eine alte Zeche begleichen, nicht über das Stadium der Spekulation hinausgedrungen. Die guatemaltekische Regierung hätte, wenn sie überhaupt zu einem Austausch bereit gewesen wäre, solche $Z$ weifel schnell durch das Anerbieten ausräumen können, anstelle der bedrohten Gefangenen andere Häftlinge freizulassen. Es ist aber nicht bekannt,

\footnotetext{
39 Ausgabe yom 1. 5. 1970.

40 Vgl. Süddeutsche Zeitung vom 13./14. 6. 1970.

41 Schon effektiver war daher die nach einer mißlungenen Flugzeugentführung von den brasilianischen Behörden an die Missionschefs gerichtete Aufforderung, in ihren Häusern zu bleiben (Die Welt v. 3. 7. 1970), wenngleich in einer solchen, den Diplomaten zur Bewegungsunfähigkeit verurteilenden Anordnung wohl nur eine vorläufige Schutzmaßnahme gesehen werden dürfte.

42 Vgl. Art. 1 und 8, abgedruckt bei F. Berber, a. a. O., S. 917.

43 In diesem Sinne auch I. von Münch, wenn er erklärt, die Grenze des Zumutbaren sei überschritten, wenn die Preisgabe zwingender rechtsstaatlicher Verfassungssätze gefordert würde, vgl. Die Zeit rom 26. 6. 1970.
} 
ob die Regierung Guatemalas einen solchen Versuch ernsthaft unternommen hat. Jedenfalls hat sie ihre Ablehnung der Forderungen der Rebellen nicht damit begründet, so daß auch insoweit das deutsche Verlangen, die Forderungen der Rebellen zu erfüllen, um das Leben Graf von Spretis zu retten, nicht als Aufforderung zu einem zumutbaren Opfer erscheinen konnte.

\section{7.}

Zuletzt bleibt noch die Frage zu erörtern, inwieweit die Haftung des Empfangsstaates dadurch begrenzt wird, daß der Entsendestaat seinerseits nicht alle ihm gegebenen Möglichkeiten ausschöpft, um seinen Diplomaten einen weitestgehenden Schutz angedeihen zu lassen. Denn allein dadurch, daß der Empfangsstaat durch das Völkerrecht zum Schutze der bei ihm akkreditierten Diplomaten verpflichtet wird, ist der Entsendestaat noch nicht der etwa bestehenden eigenen Schutzplicht enthoben. So hat Präsident Nixon die Entführung des Botschafters Elbrick zum Anlaß genommen, um beim Kongreß die Bewilligung von 1,25 Millionen US-Dollar für die Beschaffung von 33 Personenwagen zu beantragen, die mit besonderen Sicherheitsvorkehrungen ausgestattet werden sollen ${ }^{44}$. Seit langer Zeit auch haben die Vereinigten Staaten ihre Auslandsvertretungen, entsprechend deren Größe und Schutzbedürftigkeit, mit besonderen Schutzmannschaften ausgestattet, die sich aus Angehörigen einer Elitetruppe, der sogenannten Marineinfanterie, zusammensetzen ${ }^{45}$.

Einen solchen zusätzlichen Schutz, den die USA für ihre Diplomaten bereithalten, kennen jedoch die meisten Staaten nicht. Sie verlassen sich regelmäßig darauf, daß der Empfangsstaat selbst die notwendigen Schutzvorkehrungen für die fremden Diplomaten trifft. Völkerrechtlich ist daher auch allein dieser für die Sicherheit der ihm anvertrauten Diplomaten verantwortlich. Soweit eine entsprechende Haftung des Entsendestaates besteht, kann sich diese nur aus innerstaatlichen Vorschriften ergeben, im deutschen Recht beispielsweise aus der Vernachlässigung der Fürsorgepflicht, wie sie der Bund allen seinen Beamten schuldet ${ }^{46}$.

Aus der völkerrechtlichen Alleinverantwortlichkeit des Empfangsstaates für die Integrität der bei ihm akkreditierten Diplomaten folgt aber auch, daß der Entsendestaat bei seinen Bemühungen, seinerseits etwas für die Sicherheit seiner Diplomaten zu tun, gehalten ist behutsam aufzutreten. Trotz einer nach innerstaatlichen Grundsätzen gegebenen Schutzpflicht darf der Entsendestaat regelmäßig nur im erlaubten Rahmen seines außenpolitischen Handlungsfreiraumes tätig werden, das heißt, eigene Maßnahmen finden notwendigerweise im Interventionsverbot ihre Grenze. Es ist daher fraglich, ob der Entsendestaat den Empfangsstaat so unter Druck setzen darf, daß, wie es nach der Entführung des Botschafters Elbrick der Fall gewesen sein soll, der Empfangsstaat keine Entscheidungsfreiheit mehr besitzt, ob er die Forderungen der Rebellen erfüllen will oder nicht. Dagegen hält sich die an dritte Staaten gerichtete Bitte des Entsendestaates, vermittelnd auf die Regierung des Empfangsstaates einzuwirken, noch innerhalb der Grenzen des im zwischenstaatlichen Verkehr Zulässigen. Das an die Rebellen gerichtete Anerbieten des Entsendestaates, an Stelle des Empfangsstaates für die geforderte Lösungs-

44 Süddeutsche Zeitung vom 13./14. 6. 1970.

45 Die Entführung des Botschafters Elbrick wird allein dessen Sorglosigkeit zugeschrieben, vgl. Die Zeit vom 1. 5. 1970.

$46 \mathrm{Vgl}$. $\$ 79$ des Bundesbeamtengesetzes. 
summe aufzukommen ${ }^{47}$, wird wiederum nur im Einvernehmen mit der lokalen Regierung erfolgen können und ohne dieses nur in solchen Fällen, in denen der Empfangsstaat seiner aus der Schutzpflicht herrührenden Rechtspflicht zur Wiederherstellung der Integrität des entführten Diplomaten nicht nachkommt. Selbst in diesem Fall wird der Entsendestaat aber den Eindruck zu vermeiden haben, als solle die Fühlungnahme mit den Rebellen die Regierung des Empfangsstaates aus ihrer völkerrechtlichen Verantwortung entlassen - eine fatale Folge, wenn die direkten Verhandlungen mit den Rebellen scheitern sollten ${ }^{48}$.

Als Ergebnis läßt sich darum feststellen: Der ausländische Diplomat kann damit rechnen, daß im Falle seiner Entführung für den Empfangsstaat eine maximale Haftung gegeben ist. $\mathrm{Ob}$ ihm diese auch zuteil wird, hängt aber weitgehend von den bestehenden Machtverhältnissen, insbesondere von den politischen Einflußmöglichkeiten seines Heimatstaates ab.

47 So die Bundesregierung im Falle des Grafen von Spreti an ihre Botschaft in Guatemala, vgl. Weisung No. 746 und No. 1400, Bulletin des Presse- und Informationsamtes vom 21. 4. 1970.

48 So auch Außenminister Scheel in seiner vor dem Bundestag am 17. 4. 1970 abgegebenen Erklärung, vgl. Das Parlament vom 25. 4. 1970. 